\title{
Towards a Socially Adaptive Digital Playground
}

\author{
Robby van Delden \\ Human Media Interaction, University of Twente \\ P.O. Box 217 \\ Enschede, The Netherlands \\ r.w.vandelden@utwente.nl
}

\begin{abstract}
We are working towards a socially adaptive digital playground for children. To this end, we are looking into nonverbal synchrony and other social signals as a measure of social behaviour and into ways to alter game dynamics to trigger and inhibit certain social behaviours. Our first results indicate that we can indeed influence social behaviours in a digital playground by changing game dynamics. Furthermore, our first results show that we will be able to sense some of these social behaviours using only computer vision techniques. I propose an iterative method for working towards a socially adaptive digital playground.
\end{abstract}

\section{Categories and Subject Descriptors}

D.2.6 [Software Engineering]: Programming Environmentsinteractive environments

\section{General Terms}

Design, Experimentation, Measurement

\section{Keywords}

Digital playgrounds, children, social behaviour, interactional synchrony, physical activity

\section{INTRODUCTION}

The human is a social animal, we mainly talk, do, live and play together. Most children learn social abilities during the first years of their life and play tends to be an essential activity for this. At the same time we see that less social alternatives to playing on the traditional playground are becoming more and more attractive, especially playing computer games. Although one might debate about whether the amount of active play from children diminishes because of this, we should at least keep in mind that an additional incentive for social and physical active play might be beneficial. Especially if we can subtly guide ways in which children play towards more social and inclusive forms of play.

Permission to make digital or hard copies of all or part of this work for personal or classroom use is granted without fee provided that copies are not made or distributed for profit or commercial advantage and that copies bear this notice and the full citation on the first page. To copy otherwise, to republish, to post on servers or to redistribute to lists, requires prior specific permission and/or a fee.

IDC 2012 June 12-15, 2012, Bremen, Germany

Copyright 2012 ACM 978-1-4503-1007-9 ...\$10.00.
Traditional play has several important social, physical and cultural components but only rarely interactive games actively sense social activity and encourage specific aspects upon this.

In our research we will further develop and combine methods for social signal processing and actual implementations of these signals in a socially adaptive digital playground for children. On the input side this will be based on existing and ongoing work on social signal processing (e.g. see [16] for an overview), I will focus my research in the field of social signal processing to on-line synchrony detection. In specific on the non-verbal rhythmic synchrony. This unconscious adaptation of body movement to a joint rhythm with other(s) has been related to increased levels of rapport and entitativity [4]. This rapport, a measure for feeling of closeness to the other, might in turn be a key factor in social interactions on a playground. We expect we will be able, by adapting the playground, to increase and decrease levels of synchrony unconsciously and use this to increase rapport and entitativity between certain individuals or between the system and its users. It has already be shown several times that these human-human principles are applicable in the virtual domain on robots or virtual agents as well[9].

\section{DIGITAL PLAYGROUNDS}

In literature and the commercial field several examples of interactive digital playgrounds can be found. Interactive playgrounds or digital playgrounds add the interactive attractiveness of modern technologies to the playground in order to stimulate play, which in turn might target a higher end purpose including increasing physical activity, stimulating social interaction and increasing cognitive development.

On the implementation level one could make a general distinction of interactive playgrounds, according to [13] this can be done in four interactive categories: floors, walls, playground props and installations. I will use a recent set of examples to illustrate these categories combined with their mentioned higher end goals to give an idea of the possibilities of interactive playgrounds.

Wyeth et al. [17] created an interactive floor consisting of a pressure sensitive array recognising the position of the feet and a camera projecting for instance a football pitch. Several other type of games were created on the platform such as an explorative application of playing the piano, a competitive car race game and a more collaborative herding the sheep game. It mainly targeted the inclusion of participants that have a mental disability into playing interactive games and with this increasing their social interactions and 
physical activities.

The commercial system sutu is an interactive wall for playing football outdoors, it has a grid of pressure sensitive glass, sound feedback and bright coloured LED squares [18]. Several games are implemented requiring to hit the wall with a non-equipped football within a certain time and or to hit a certain area. Mentioned higher end goals are social development, healthy exercise and neighbourhood involvement [18].

Rosales et al. [10] developed a simple playground prop, consisting of a shoe equipped with two pressure sensors. When child jumps with these shoes a sound is played and a LED light placed on the shoe is turned on . Their targeted higher end goal focused on increasing social skills by encouraging free-play. Often these kind of playground props can be used everywhere and are not restricted to a certain area.

Soler et al. [12] created an interactive installation consisting of an inflatable slide with an interactive projection. Children could for instance create robots by collecting robot parts on their way down the slide in a collaborative way. Opposed to earlier examples the interactive slide is an example of my personal interpretation of an interactive installation: an interactive installation constitutes an entire interactive playground environment on its own, opposed to earlier examples introducing an interactive element into an existing environment or game. The interactive slide was created with increase of social activity and physical activity mentioned as higher end goals.

Although researchers often only provide examples of developed interactive playgrounds and some initial findings, more comprehensive research frameworks [11], design methods [14], guidelines [1] and terms to analyse these playgrounds [6] can also be found in recent research.

\section{SYNCHRONY MEASURES}

Several automatic detection measures for synchrony have been developed for both audible and visual cues. Only using computer vision technologies will simplify the problem of detecting synchrony and social signals when compared to an approach using both cues. Two main differences are the dimensionality which is much higher for unprocessed video and the sample rate which is in the domain of approximately several $\mathrm{kHz}$ for speech and typically only several frames a second for visual cues. In a comparability study of audio and visual features used for synchrony measures [2, Table 1.a] it can be seen that in a cooperative task the visual synchrony cues have a higher rate of significant correlations than correlation between visual and audible cues, although this might partially be due to the fact that roles in the given task result in disproportional amount of speech. Even though the authors actually argue the multi-modality of synchrony, their results thus seem to indicate that comparing visual cues to each other might be an effective measure. Furthermore our recent observational research of children playing at an interactive playground using a newly developed annotation scheme showed possibilities to reliably detect social behaviours solely using visual cues.

One problem when dealing with synchrony is to account for synchrony by chance. As we all have our own natural bodily rhythms [3] and random movements these will correlate every now and then without any specific meaning. However when these movements line-up more frequently or mutually adapt to each other (entrainment) than we have a more interesting phenomena. One way to account for ran- dom synchronisation in a session is to apply Rammseyer's bootstrap method [7]. In his method every time slot is not only compared to movement of the other person at that surrounding time frame but is also compared to a base line of 100 randomly selected time slots. In this way we get an overall view per session in which one can differentiate a significant number of synchronous movements from synchrony by chance.

Although this and several similar approaches have been proposed and showed effective for evaluation afterwards, we are especially interested in ways to measure synchrony directly and at a specific time. We won't have enough data in the beginning of the session to compare to 100 other time slots to counter balance for randomness and we want to have a measure for an amount of synchrony occurring at that time slot opposed to that occurring in an entire session. To this end we see possibilities in using more intelligent and robust ways of measuring body movement. For instance Kinect and technologies alike provide an easy-to-use and quite robust measurement. Using this information we can then also add weighing factors for certain movements like head sway, face scratching, upper body sway and foot movement, as we might find that some movements contain more relevant information about movement synchrony than others.

At the current moment we are unaware of any proper online measure of synchrony that sufficiently deals with filtering for random synchrony occurrences, nor are we familiar with any application of synchrony measures in an interactive playground. Such an on-line measure will give us usable information for our interactive playgrounds. For instance, upon detecting high synchrony levels between certain individuals we might gradually change game dynamics in such a way that other individuals will be tempted to work together, e.g. by introducing a common (virtual) enemy. This and similar interventions could in turn be beneficial for diminishing bullying, increasing physical activity and increasing social awareness and capabilities.

\section{RESEARCH TOPICS}

The research topics I will be working on deal with both social signal processing and development of adaptive interactive playgrounds for children. For creating an on-line measure of non-random movement synchrony bursts we need to develop alternative method for measuring movement synchrony. This will require training data, proper selection of manual observation methods that will function as ground truth measurements and creating computer vision implementations to test these created synchrony detection methods. We need to compare several measurements of the children in the playground that could be used for measuring synchrony like the proximity, position and weighted or nonweighted posture changes. At the same time we need to develop an interactive playground in an efficient way. This interactive playground has to be adaptive using sensed social signals. In order to reach this goal of creating a socially adaptive interactive playground we will have to find relations between the game dynamics and social behaviour, for example what social behaviour are likely to following from possession of an object or ability to destruct something in the game. Furthermore ways to unconsciously make and break non-verbal synchrony and the reported link to rapport, entitativity and forms of empathy in an interactive playground will be a major factor in my research. 


\section{PROGRESS TO DATE}

The most important work I have been doing so far with respect to the socially adaptive interactive playground is the development of an observation scheme for interactive playgrounds. The interactive playground used to create and test this scheme was already developed before our research started, it targeted to stimulate competition and collaboration using an interactive floor projection that reacted on position of the children as well as on movement of shaking a ball. Our observational study using recordings of play on this playground showed that with triggering competition another social behaviour is triggered as well being aggression/physical contact, thus this indicates that we indeed might be able to influence social interactions by changing game dynamics. Qualitative results of this observational study showed as well that by providing an interactive system we can let people interact with and through it, a similar effect was found by Morrison as well [6]. More importantly we showed that we can identify certain social signals reliably by only looking at them thus we might limit our selves to the visual domain for input of social signals. Probably we might even limit ourselves to skeleton tracking of persons in the space, which reduces dimensionality of the problem drastically. This feeling is strengthened by first implementations of using the Kinect and by closely following student-projects implementing this technology, this showed that it provides a great platform to do real time posture measurements in an easy way.

Our current interest for interactive playgrounds is to create open-ended play, meaning we like to create an explorative experience instead of creating a game accompanied with giving explicit instructions and goals. Although there are several research papers about open-ended interactive playgrounds, we still need to gain a deeper insight in game dynamics in such an environment to stimulate and inhibit certain social signals. For example in addition to factors mentioned elsewhere we found that it might be beneficial for the entertainment level to have at least some destructive feature. This destructive feature should also be turned on or off to elicit or reduce competitive behaviour and therewith physical contact (as found in our observations of the interactive playground), especially because several projects indicated that children and adults seem to enjoy destroying things in the virtual domain as will be described next. In demonstrators of an interactive bar [8] we repeatedly witnessed users intentionally trying to smash its virtual inhabitants. During a therapeutic game children especially liked hitting and thereby destroying a target [15]. In the interactive playground children were especially busy shaking a ball after it was explained that this resulted in releasing poison, which in turn would have a destructive effect for others, as well as stumping on objects in an attempt to destroy these [14].

Currently we are preparing to take recordings of children playing in an uninstrumented playground to find cues for social behaviours to create automatic detection systems for these behaviours.

\section{RESEARCH METHODS \& AGENDA}

As stated before we will record children's play and we will create a corpus of children's play with this to use as a baseline measurement, to find appropriate measurements for sev- eral social behaviours and to use as training data. We need to create on-line measurements for social signals that act as robust as possible on several children at a time. For synchrony this will require several comparative studies. We will amongst others compare the to be created on-line methods to Ramseyer's off-line bootstrap method as well as to manual annotations for more temporary measures. We will compare and apply several existing techniques to the domain of synchrony measures, like person and skeleton tracking, proximity measures and looking into significance of synchrony of certain posture changes like body sway, arms crossing and face touching.

In the domain of analysing children's play it is not yet straightforward what constitutes a certain level of physical activity and the current golden standard to measure physical activity seems to be pedometers [5]. Although this might work, measuring from a distance as with the Kinect without attaching devices to the participants is beneficial in several situations, for instance it is less obtrusive and is therefore less likely to influence test results. Another advantage is that weighted physical activity might give better indication of actual physical activity. Concluding, we might take this into account in the first recordings of our corpus resulting in a side product of our endeavours that might be useful in other research areas. This kind of realisations that research might be applicable to other areas should be kept in mind in the entire research.

Most importantly we will have to elicit certain social signals with our adaptive playground. We will need to research game dynamics and social interactions in implementations of several versions of playgrounds for this, to tailor it and to find reusable effective methodology. To have such an efficient way of developing several versions of interactive playgrounds we build upon the several methods, frameworks, terms and guidelines that already exist. One approach for a design platform developed by Rozendaal et al. [11] seems to be very appropriate. I have a similar approach in mind, which I will explain with my own terms. This is probably based on several other already existing techniques and a similar approach is used in [14].

Most systems, even when open-ended play is targeted, have a higher end goal like improving social behaviour or stimulating physical activity that is not explicitly made clear to its users. In order to reach this goal we need some entertaining interactions. The second level I call the rationale and bridges the interactions and higher end goals, it is the explanation why a certain interaction would elicit this higher end goal, preferably based on sound research findings. The third level is a set of interactions describing a set of user actions and system responses. The fourth level is the implementation and contains hardware and software descriptions as well as the visualisations which in turn may benefit from a rationale as well. A metaphor can be chosen to create a platform for easily developing the interactions, graphics and rationales. In some occasions the system is developed systematically from top down but often either certain interactions or a entire system are thought up and implemented and only later on accompanied with other interactions to fulfil a certain higher end goal that arises during the process. During the development of the socially adaptive interactive playground these steps will be recorded to show that a usable design technique for such interactive systems can arise using these levels. 
We will need several iterations on developing interactive playgrounds and experimentation in ways to induce synchrony. For convenience we might use target groups that can be approached more easily in some experiments where children are not explicitly needed. In general we will target children at the end of primary school being eight to twelve years old, as these already developed a form of synchrony, are still inclined to explorative playful interactions and during evaluations will have less problems communicating their thoughts when compared to other age groups of children.

\section{CONCLUSION}

We are working towards a socially adaptive interactive playground. We are researching ways to create an on-line synchrony measurement to use in this context. We seem to be able to achieve our goal of creating such a socially adaptive playground using computer vision techniques for detecting some of these social signals. This expectation is based on our first findings, these showed that at least some social behaviour is the indirect result of interactions in an interactive playground and at the same time we are able to identify such social interactions with visual cues only. Actually having a socially adaptive playground could be a way to counteract the increasing fear of society that modern technologies result in more and more social interaction problems in current youth and that having these modern technologies results in children of today having less physical activity. During this research several other useful products are likely to arise such as effective design and evaluation techniques, insight in game dynamics on entertainment and an automatic measurement of physical activity.

\section{ACKNOWLEDGMENTS}

This publication was supported by the Dutch national program COMMIT.

\section{REFERENCES}

[1] T. Bekker, J. Sturm, and B. Eggen. Designing playful interactions for social interaction and physical play. Personal Ubiquitous Comput., 14:385-396, July 2010.

[2] E. Delaherche and M. Chetouani. Multimodal coordination: exploring relevant features and measures. In Proceedings of the 2nd international workshop on Social signal processing, SSPW '10, pages 47-52. ACM, 2010.

[3] S. Gill. Rhythmic synchrony and mediated interaction: towards a framework of rhythm in embodied interaction. AI \&S Society, 27:111-127, 2012.

[4] D. Lakens and M. Stel. If they move in sync, they must feel in sync: Movement synchrony leads to attributed feelings of rapport. Social Cognition, 29:1-14, 2011.

[5] P. D. Loprinzi and B. J. Cardinal. Measuring children's physical activity and sedentary behaviors. Journal of Exercise Science E3 Fitness, 9:15 - 23, 2011.

[6] A. Morrison, S. Viller, and P. Mitchell. Building sensitising terms to understand free-play in open-ended interactive art environments. In Proceedings of the 2011 annual conference on Human factors in computing systems, CHI '11, pages 2335-2344. ACM, 2011.
[7] F. Ramseyer and W. Tschacher. Synchrony: A core concept for a constructivist approach to psychotherapy. Constructivism in the Human Sciences, 11(1):150-171, 2006.

[8] D. Reidsma. Anemone - an interactive light projection, September 2009. Demonstration at Discovery'09, NEMO in Amsterdam, the Netherlands.

[9] D. Reidsma, A. Nijholt, W. Tschacher, and F. Ramseyer. Measuring multimodal synchrony for human-computer interaction. In A. Sourin, editor, Proceedings of the International Conference on CYBERWORLDS 2010, pages 67-71. IEEE Computer Society Press, October 2010.

[10] A. Rosales, E. Arroyo, and J. Blat. Feetup: A playful accessory to practice social skills through free-play experiences. In INTERACT (3), pages 37-44, September 2011.

[11] M. Rozendaal, A. Vermeeren, T. Bekker, and H. de Ridder. A research framework for playful persuasion based on psychological needs and bodily interaction. In A. Salah and B. Lepri, editors, Human Behavior Unterstanding, volume 7065 of Lecture Notes in Computer Science, pages 116-123. Springer Berlin / Heidelberg, 2011.

[12] J. Soler-Adillon and N. Parés. Interactive slide: an interactive playground to promote physical activity and socialization of children. In Proceedings of the 27th international conference on Human factors in computing systems, CHI EA '09, pages 2407-2416. ACM, 2009.

[13] J. Sturm, T. Bekker, B. Groenendaal, R. Wesselink, and B. Eggen. Key issues for the successful design of an intelligent, interactive playground. In Proceedings of the 7th international conference on Interaction Design and Children, IDC '08, pages 258-265. ACM, 2008.

[14] D. Tetteroo, D. Reidsma, B. van Dijk, and A. Nijholt. Design of an interactive playground based on traditional children's play. In Proceedings 4th International ICST Conference on Intelligent Technologies for Interactive Entertainment (INTETAIN 2011), May 2011.

[15] R. W. van Delden. Design of therapeutic TagTile games for children with unilateral spastic cerebral paresis. Master's thesis, Human Media Interaction, University of Twente, September 2011.

[16] A. Vinciarelli, M. Pantic, and H. Bourlard. Social signal processing: Survey of an emerging domain. Image Vision Comput., 27:1743-1759, November 2009.

[17] P. Wyeth, J. Summerville, and B. Adkins. Stomp: an interactive platform for people with intellectual disabilities. In Proceedings of the 8th International Conference on Advances in Computer Entertainment Technology, ACE '11, pages 51:1-51:8. ACM, 2011.

[18] Yalp InterActIve. Sutu: Interactive sports wall. http://www yalpinteractive.com/10-Home.html, 2012. Last accessed February 21, 2012. 